\title{
Line cluster detection using a variant of the Hough transform for culture row localisation
}

Leemans, V.; Destain, M.-F.

Gembloux Agricultural University - Unité de mécanique et construction

Passage des Déportés, 2 - B 5030 Gembloux, Belgium

leemans.v@fsagx.ac.be

\section{Abstract}

An adaptation of the Hough transform was proposed for the detection of line clusters of known geometry. This method was applied in agriculture for the detection of sowing furrows created by a driller and of chicory plant rows during harvesting process.

The sowing rows were revealed by a background correction, the background being obtained thanks to a median rank filter. The method was found efficient in eliminating the shadows. For the crop rows, a neural network was used to localise the plants. While the petiole and the leaves were easily separated from the soil, the chicory root and the soil having about the same colour and the lighting condition varying widely, it was more difficult to obtain a good contrast between those parts, which leaves place for some improvements. The adapted Hough transform consisted in computing one transform for each line in the cluster with, for reference, the position and direction of the theoretical position of the row. The different transforms were then added. It was found effective for both the sowing rows and the chicory rows. Results remained good even in very noisy conditions, when the rows were incomplete or when artefacts would lead its classical counter part to show several alignments other than the expected ones. The culture rows were localised with a precision of a few centimetres which was compatible with the proposed applications.

Keywords : Hough transform; line cluster; guidance; crop rows 


\section{Introduction}

\subsection{The problem identification}

Automatic detection of line clusters could find many applications in agricultural machines. Guidance of inter-row hoeing, sowing or harvesting operations are some possible uses of this technique. This paper focuses on the guidance assistance of a precision drill for sugar beet or chicory relative to previous rows on one hand and on the harvest of chicory on the other. The drills are wide machines of usually twelve or eighteen rows width which are actually fixed rigidly to the tractor's three-point hitch and the position of the drill relatively to the previous rows is controlled by the driver. This imposes that the harvesting machines have a width being a submultiple (usually six) of the drill's width and that it follows several months later the path of the drill, which presents an evident lack of flexibility. To encompass these limitations (to enable the use of eight row harvesting machines), deviation from the standard row interval between lines sowed by successive passages should be less than $0.15 \mathrm{~m}$. On the other hand, guidance of chicory harvesting machines is a difficult task that imposes manual steering and constant attention of the driver. In this case, a higher accuracy is required and misalignments higher than $0.05 \mathrm{~m}$ should be avoided.

These applications have in common the necessity to detect multiple lines or rows of plants. The Hough transform is widely used for localisation of linear objects in an image. This transform is quite robust against noise, missing parts etc., but limitations can be encountered when the noise becomes important compared with the contrast of the objects and when lures are present in the images. In these situations, the detection of the maxima corresponding in the Hough space to the rows in the image could become a difficult task. The studied application presents those characteristics. Indeed, in the detection of sowing rows, variations in the soil's relief induce differences in the irradiance and consequently clods or tillage furrows add high uncertainty. At harvest stage, the chicory leaves cover most of the ground and the row localisation could only be achieved after the leave stripper topper. On a properly topped root the leaves and normally the petioles are gone, leaving the collar and the 
root. The guidance must then be based on plant elements having a colour close to the background colour. The uncontrolled lighting conditions contribute to increasing the variability. The use of a method based directly on the classical Hough transform in those high uncertain conditions would cause a lack of robustness. On the other hand, the field environment is well structured, for example the inter-row distance is known and is constant for a given machine. An adaptation of the transform is proposed in this paper to make it more robust for the detection of line clusters by taking into account the a priori geometrical knowledge. Indeed, the lines being sowed by one passage of the drill, their orientation and spacing remain constant respectively to each other. The position of the tool relatively to these lines is however unknown. The transform was adapted so that the maximum of each line was added at the same point of the Hough space. The effect is to concentrate the maxima belonging to the cluster and diluting the others. The position of this maximum was strictly related to the position of the tool respectively to the lines. The Hough transform being sensitive to shadows, these should be removed. A pretreatment was proposed for each application, in order to obtain bright rows on a dark background.

\subsection{Bibliography}

Different authors addresses the question of how to extract the data from the images, to obtain information relevant for machine guidance assistance or for autonomous vehicles. The problem of dealing with natural lighting should also be considered in the literature review.

Different approaches of the problems are proposed, such as the Hough transform, the determination of the vanishing points or a band strip approach. Pla et al. [1] based their algorithm on the detection of the vanishing point of the row cluster. This technique was borrowed from road vehicle guidance and the authors stated that compared with other applications, the "natural" environment is characterised by irregular and undefined shapes with a high texture content. The processing of the images included the segmentation of the colour images, the computation of the rows skeletons, the localisation of straight lines, the tracking of the vanishing point and the extraction of information 
from the lines concurring to the vanishing point. However this technique seemed highly computational demanding. Marchand [2] described a method to track rows of various widely spaced cultures (tested on cauliflowers, sugar beet and widely spaced double rows of wheat). The original images of plant rows were segmented by "thresholding" and the objects were represented by points and weighed according to their surface. The position of the central row was localised in the Hough space in a small window centred according to the expected position of the row. Thus only one row was taken into account for the localization. The typical error was $18 \mathrm{~mm}$ and the images were treated at a rate of 10 images per second. Tillett et al. [3] proposed a method for guidance of inter-row hoeing in sugar beet fields. Authors exploited the periodic variations of luminance between the soil and the plants due to the parallel crop rows, on horizontal scan lines extracted from the images. A filter was applied which allowed the frequency of the crop rows to be extracted whilst attenuating the lower frequencies effects of shadows and higher frequency due amongst other to weeds. The standard deviation of the error relative to crop row was below $16 \mathrm{~mm}$. Søgaard and Olsen [4] used an original method for double spaced wheat row localisation. The grey level images were divided into horizontal strips and then each strip into sub-strips having a width corresponding to the inter-row width. The gravity centre of the mathematically enrolled mean sub-strip gave, for each strip, the position of the rows and an estimation of the relative accuracy. The estimation of the offset and of the orientation was given by weighted linear regression for the image. Authors stated that this procedure was chosen to reduce the computational time. The precision varied from 6 up to $12 \mathrm{~mm}$ depending on the development of the crop. Rovira-Más et al. [5] detected two crop rows (soy beans) in five steps including the definition of a region of interest (to limit the computation load), the image binarization using an adaptive threshold, the research of the midpoints of the rows, the computation of the Hough transform and a connectivity analysis. The Hough transform returned several maxima each corresponding to an alignment in the image. When two maxima were present it was expected that each represent the position of a row and the middle point was used to evaluate the tractor's relative position with respect to the two rows. Otherwise, the algorithm was adapted: when one 
maxima was present, it was supposed that the two observed lines were recovering each other (plants at an advanced stage) and its parameters were used directly ; for more lines, a threshold distance between the maxima in the Hough space were used to cluster them. This shows the difficulties encountered with the classical Hough transform.

The main problems encountered in outdoor image acquisition are the unevenness of the lighting conditions in time and space. This problem is particularly accurate for methods segmenting the images ([2], [5], [6]). If the unevenness in time could be partially compensated by the camera itself or by adjusting threshold levels with the mean grey level of the image (as in [5] and [6] for example) or by developing robust algorithms ([3], [4]), the unevenness in space should be considered carefully. Empirical shadow compensation algorithms were developed by Pilarski et al. [7], while Onyango and Marchand [8] proposed a RGB (red, green, blue) image to grey level image transform to eliminate the effect of the shadows, computed thanks to the spectral characteristics of the illuminant, of the soil and of the plants.

Amongst the methods proposed to localise the rows, those described by Pla et al. [1], Tillett et al. [3] and Søgaard and Olsen [4] would require sufficiently wide and continuous rows in order to work properly, conditions which were not both fulfilled in the applications considered in this paper. The traces left by the drill are almost continuous, but quite thin and the chicory rows, though wide, showed a frankly discontinuous aspect. The method applying a threshold ([2], [5], [6]) needs a good contrast between the target and its surrounding. The poor contrast and the many lures encountered in the drill traces' detection would prevent the use of such algorithms in this application. The wide colour variability in the element constituting the chicory rows (detailed below) could not allow the use of methods such as proposed by Pilarski et al. [7] or Marchand [8].

In conclusion, the method to be developed should work in poorer contrast conditions and higher noise, compared with the method applied in outdoor conditions previously described. 


\section{Material and method}

\subsection{Image acquisition}

Video sequences were acquired during Spring 2003 and 2004 for drilling and during Autumn 2003 for harvesting. An inexpensive USB camera Philips PCVC 740K (Koninklijke Philips Electronics N.V., Eindhoven, the Netherlands) was used in 2003. A Unibrain Fire-iA00 1394 camera (Unibrain S.A., Athens, Greece) equipped with a lens having a focal of $6 \mathrm{~mm}$ was used in 2004. Both were colour mono-CCD cameras. The aperture was fix for the USB camera and was set manually for the Unibrain camera. The automatic settings of the electronic shutter were found suitable for the application.

The video sequences were saved as AVI files. Table 1 gives the number of places and the number of videos acquired each year and for each season (drilling or harvest). Thanks to these videos, the methods were tested off-line, but in real time.

\subsection{Pretreatments}

The classical pretreatment of the Hough transform consists in revealing the boundaries of the objects and applying a threshold in order to obtain bi-level images. The aim of this research was to localise linear elements rather than to find the boundaries of objects. Consequently the pretreatments did consist in an edge detection, but in making the lines visible and removing unwanted objects such as the shadows. The results of the pretreatments were grey level images, not bi-level images.

The management of the videos and of the images, the image filtering were made by using the Open Source Computer Vision Library (Intel Corp.). The codes of the adapted Hough transform and the post filtering were written in $\mathrm{C}++$ using gcc (Free Software Foundation, Inc.).

\subsubsection{Detection of sowing rows}

The camera was fixed on the machines outside the left extremity of the drill, in such a way that at 
least two rows could be observed.

For our concern, the essential part of a sowing element are a coulter and a weighting wheel framed by two supporting wheels, as shown in Fig. 1. This setting leaves a flat profile of about $0.06 \mathrm{~m}$ width, with a small hollow in its centre (Fig. 1). For each row, the central hollow as well as one boundary of the flat profile were usually visible on the images as dark lines on a bright background, as can be observed in Fig 2.

The rows differ from the rest of the ground only by their intensity and a detection method based on the hue could not be used. In this case, the main problem was to get rid of the shadows possibly present on the images. The clods and the many lines resulting from the tillages also induced an important noise. The former problem was addressed by the pretreatments while the latter one was overcome by the pretreatment, by the Hough transform itself and by a subsequent filtration.

Practically, as the colour did not seem to be relevant for this application, only the green channel was used. The full resolution was not required and a size reduction was operated. Part of the noise due mainly to clods was removed by using a Gaussian filter. As the noise had no privileged orientation while the rows were almost vertical, distortions were introduced in both treatments. The size of the image was reduced from $320 * 240$ pixels to various sizes from $120 * 90$ pixels to $60 * 60$ pixels. Square and rectangular Gaussian filters of various dimensions were also tested.

The shadows and any other unevenness were removed by a background subtraction. It consisted in computing a background image by using a wide median rank filter and in subtracting the image from the background, which revealed the row as a bright line on a dark background, as shown in Fig. 2 . The median filter is well suited to remove noise spikes and thin lines while leaving the edge of the objects non blurred. A linear filter could not produce the same effect. The width of the filter, related to the size of the image is the important parameter of this algorithm. The tested area varied from $3 * 3$ pixels up to $11 * 11$ pixels. This method was described in detail by Leemans and Destain [9]. 


\subsubsection{Detection of chicory rows during harvesting}

The camera was placed between the leaf stripper topper and the harvest shares. The scene presented in Fig. 3 shows, apart from the soil (brown), parts of the machine (red wheel rim and dark tyre), chicory roots (light brown, more orange than the soil), petioles (green), root flesh (whitish). Occasionally parts of leaves ripped off the roots were thrown between the rows. Chicory outside the rows or weeds were also encountered in some places. All the chicory parts were not visible on each image. The images in Fig. 3 were chosen to represent most of the different conditions on few images, but often, the rows in the images can be made only of plants representing some kind of the variability showed in Fig. 3. This implied that every part should be detected. Only a few plants were missing. The lighting condition could be sunny to cloudy which means that the images could show sun and shadow areas or a more or less homogeneous illumination.

To reveal the plant rows in such a variability, a neural network was used to detect pixels belonging to the ground from those belonging to a chicory. It consisted in a two layer perceptron with one hidden layer. Seven inputs were considered : the luminances of the pixel in each of the R, G, B (red, green and blue) channels, the mean luminance of those channels for the whole image and the standard deviation of the green channel for the image (providing an information about the contrast). The computation of the statistical parameters was based on a systematic sub-sampling of the image (one pixel out of twenty-five). Sigmoidal transfer function were used for both layers of the perceptron.

The training of the neural network were carried out off-line (using Matlab, The Mathworks, Inc.) The data were extracted from the videos by an operator thanks to a program written to select a set of data with the mouse, on one out of six videos from each plot, and on one image each second (thus one out of thirty images). The main drawback of the neural network is its poor ability in extrapolation. As an attempt to test the robustness of the neural network, the data from one plot was divided randomly into three sets (one half as learning set, one quarter as stop set, one quarter as validation set) and the data from the other plot were used for validation only. 
After training, the neural network was implemented with the other image treatments. The images extracted from the videos and were resized to the third of their original size (106 pixels wide and 80 pixels high). After having computed the required statistics on the image, the data of each pixels were fed into the neural network which were returned values between 0 and 1 . These values were multiplied by 255 and stored in one byte per pixel. The result applied to the images of Fig. 3 is presented in the first row of Fig. 6. The plant rows appear in bright while the ground and the machine remain darker.

\subsection{Adaptation of the Hough transform for cluster lines detection}

The Hough transform was designed to detect curves in images, amongst other straight lines. Though chicory rows do not appear as continuous lines, the alignment of plants could also be detected with this method.

As stated amongst other by van der Heijden [10], a straight line can be represented as an equation of the angle $\alpha$ of the line with a reference direction and of the distance $r$ to a reference point, according to Fig. 4 (upper left) :

$$
\left(x-x_{r e f}\right) \cos (\alpha)-\left(y-y_{r e f}\right) \sin (\alpha)-r=0 \quad \text { with } \alpha \in[0, \pi], \quad r \in \mathbb{R}
$$

The reference point is usually the centre of the image and the reference direction the horizontal. A line in the $(x, y)$ plane is thus represented as a point in the $(\alpha, r)$ plane (the Hough space - Fig. 4, upper right $)^{1}$. To search the position of that point, the integral of the image perpendicularly to a projection line crossing the centre of the image and having the given angle with the reference direction can be computed. This has to be done for several angles and is usually represented as a transformed image, with the angle in abscissa and the position along the line relatively to a reference point in ordinate. A bright line on a dark background appears as a maximum on the transformed image (Fig. 5, line 2).

1 And a spot in the (x,y) plane is represented by a sine in the ( $\alpha, \mathrm{r})$ plane, as can be seen in Fig. 5 and 6. 
When the camera observed the crop rows, several ones were visible in the image as shown in Figs 2 to 4 . On the ground, the culture rows are parallel with a given spacing and appeared in the images as almost straight and sequent lines. The spacing and orientation of these lines in the image depend on the geometry of the image acquisition (focal length, CCD dimensions, tilt of the camera). We made the assumption that all those lines converged to the same point (out of the image, Fig. 4) and that the angle and the distance between these lines remained constant when the machine (and thus the camera) offset or changed its direction relatively to the previous sowing traces or to the plant rows. The angles and distances were thus related by the following equations :

$$
\begin{gathered}
\Delta x_{i-j}=\frac{-r_{i}}{\cos \left(\alpha_{i}\right)}+\frac{r_{j}}{\cos \left(\alpha_{j}\right)} \\
\theta_{i-j}=\alpha_{i}-\alpha_{j}
\end{gathered}
$$

When the camera moved with reference to the culture row, the lines in the images and their corresponding maxima in the Hough transform image also moved, but their relative positions remained unaffected.

In order to detect the cluster of lines in one operation, one transform was carried out for each line, but with different angular and lateral references, so that the maximum representing each line was positioned at the same point of the Hough space, as shown bellow in Fig. 4. The Hough transform was then given by :

$$
\left(x-x_{\text {ref } i}\right) \cos \left(\alpha-\alpha_{i}\right)-\left(y-y_{\text {ref }}\right) \sin \left(\alpha-\alpha_{i}\right)-r=0 \quad \text { with } \alpha \in[-\pi / 2, \pi / 2], \quad r \in \mathbb{R}
$$

The reference points were chosen at the intersection of the horizontal median of the image and the considered (the $\mathrm{i}^{\text {th }}$ ) reference lines ( $\mathrm{y}_{\text {ref }}$ was thus constant). The reference angle was the one of the perpendicular to the considered reference line (Fig. 4, bellow). The number of reference lines, points and angles were equal to the number of rows in the line cluster (varying from two to four). The values of these references depended on the geometry of the acquisition (height and tilt of the camera) and were thus measured off-line, in an image of the tool (sowing machine or harvest machine) placed 
at the correct position. The transforms were carried out for angles comprised in an interval of 0.6 radian centred on the reference angle and for a width given by :

$$
w_{H T}=w_{i} / n_{\text {row }}+10
$$

with $\mathrm{w}_{\mathrm{HT}}$ the width of the part of the image to which the Hough transform was applied, $\mathrm{w}_{\mathrm{i}}$ the width of the image and $\mathrm{n}_{\text {row }}$, the number of rows in the cluster. This ensured that the whole image was covered almost once and that a row cut by the edge of the image, as the most left row on image Fig. 5 was taken into account.

The values of each part of the transform were summed. The maxima corresponding to the culture row were added, while others peaks resulting for example from the tillage, ripped leaves or weeds were added to the noise and thus diluted. When the sowing machine was correctly positioned, the coordinate of the maxima should be in $(0,0)$ (which is the centre of the image - Fig. 4, bellow, right).

\subsection{Signal filtering}

The maximum of the Hough transform gave an angle and a distance from the reference. Some noise was observed and he data were treated using a recursive low-pass filter. The filtered position vector $\left(\alpha_{\mathrm{f}}, \mathrm{r}_{\mathrm{f}}\right)^{\mathrm{T}}$ was given by :

$$
\left(\begin{array}{l}
\alpha_{f} \\
r_{f}
\end{array}\right)_{k}=\left(\begin{array}{l}
a_{\alpha} \\
a_{r}
\end{array}\right)\left(\begin{array}{l}
\alpha_{m} \\
r_{m}
\end{array}\right)_{k}^{T}+\left(\begin{array}{c}
1-a_{\alpha} \\
1-a_{r}
\end{array}\right)\left(\begin{array}{l}
\alpha_{f} \\
r_{f}
\end{array}\right)_{k-1}^{T}
$$

where k reference the image being treated and k-1 the previous image, $\left(\alpha_{\mathrm{m}}, \mathrm{r}_{\mathrm{m}}\right)^{\mathrm{T}}$ the position vector measured on the image. The coefficient $\mathrm{a}_{\alpha}$ and $\mathrm{a}_{\mathrm{r}}$ were adjusted, based on previous experience.

\subsection{The reference}

A reference was established by using a dedicated program which recorded the abscissa of the mouse while playing the videos. This was used to record the position of the bottom of the most right row in each image of the videos (its abscissa $\mathrm{x}$ according to the notation in Fig. 4, expressed in mm). 
The program treating the videos extracted each image sequentially, localised the row, computed the deviation (the absolute difference) and the difference between the reference and the corresponding point deduced from the filtered data and recorded the deviation against the elapsed time from the beginning of the video. The classical error parameters, the trueness, given by the mean of the deviation, and the precision given by the standard deviation of the difference were computed for each video. The extreme values indicating when the algorithm failed, were characterised by the third quartile and by the maximum.

Each parameter was summarised for the whole set of videos by its mean and its standard deviation.

\section{Results}

\subsection{Pretreatments}

\subsubsection{Detection of sowing rows}

Few parameters had to be fitted. The size of the reduced image were of 120 pixels wide and 90 pixels high, conserving the original width to height ratio. The lines to be detected were sometime quite thin and smaller image size tended to lessen the results. The Gaussian filter size was of 11 pixels height and 3 pixels width. This asymmetry contributed to reduce the noise while preserving the thin lines. The median filter was square and of 5 pixels. A bigger median filter led to attenuate wider objects in the background image and thus preserving them better in the final image. This was favourable to reveal the researched lines but also for other lines resulting from the soil tillage for example. These latter appeared often wider on the images and the parameter fitting led to median filter size relatively small for this application.

The results of the pretreatments are shown in Fig. 5. Most of the shadows were removed by the background subtraction. Only a thin part of the tractor's door remained. This had no consequence, because the orientation of the line drawn by that element on the image had a direction which could not be confused with the researched objects. This also means that vertically elongated objects should 
be avoided near the camera, because they could produce shadows like the sowing lines. The contrast which, on the original image, was less important in the shaded part, remained smaller in those parts of the pretreated image. Nevertheless, the lines were visible in that part too. The seed lines often appeared as thin double lines (a central hollow and a shadow of one border). One of these was detected by the algorithm which could produce a slight bias.

\subsubsection{Detection of chicory rows}

Only a set of five parameters were kept (R, G, B and the means for R and B) to feed the neural network. Three neurons were necessary in the hidden layer. The ratio of data correctly classified was around 70\% (this is for the validation data, the learning and stop set presented usually a higher rate, around 80\%). The first row of Fig. 6 show the results for the images of Fig. 3 . Other configurations such as a neural network fed with the seven parameters and without hidden layer were found to have higher correct classification rate (above $80 \%$, for the three sets), but the errors were systematically made in the shadow or in the sunny part of images, when both were present, such as in the fourth line of Fig. 3. Usually the chicory was not revealed by the network in those parts of the image. With this neural network, on the contrary, the contrast between the plants and the soil was less important, but there was almost always a contrast, even if the uncertainty remained important between some parts of the roots and of the soil (Fig. 6-1d).

\subsection{The adapted Hough transform}

The images in the second rows in Fig. 5 and 6, representing the result of the classical Hough plane for angles varying from $-\pi / 2$ up to $\pi / 2$ rad, are given for didactic purposes and were not used by the proposed algorithm. The image of the third row (Fig. 5 and 6) were the results of the adapted Hough transform (for angles varying from -0.3 to 0.3 radian around the reference). For both the classical and the adapted Hough transform representations, the grey scale was scaled so that the black level represents the minimum and the white level represents the maximum. 
In good conditions when the rows were clearly visible, as in Fig. 5-a and Fig. 6-a, one maxima was observed for each crop row for the classical Hough transform. The detection of these maxima might have been achieved using for example the mean-shift. When the conditions were less favourable, the maxima decreased while others might appear. This can be seen particularly in Fig 5-2b where highest maxima corresponded to the furrow between the sowing lines and in Fig. 6-2b, where the presence of a plant outside the row and the missing plants in the row made other alignments more visible. The problem of the association of the pairs or triplets of maxima corresponding to the lines in the cluster would became more intense. On the adapted Hough transform, one main maximum was observed in most conditions. In the case of Fig. 6-3d, the contrast between the maximum and its surrounding area was lesser which caused a certain instability and the deviation with the reference might be higher. This was however corrected by the post filtering.

Because of variations into the soils stiffness and of variations within its relief, the heigh and the tilt of the camera with respect to the traces could vary slightly. These variations, which conducted in changes in the values of $\Delta \mathrm{x}_{\mathrm{ij}}$ and $\theta_{\mathrm{ij}}$, were observed to remain feeble and their effect on the precision could not be precisely determined. The expected effect was to blur the maximum in the Hough space. As the lines to be detected had more than one pixel width and the maximum in the Hough space was consequently not sharp, which in contrast allowed a certain robustness relatively to changes in the values of $\Delta \mathrm{x}_{\mathrm{ij}}$ and $\theta_{\mathrm{ij}}$.

\subsection{Post filtering}

Considering the filtering of the signal resulting from the image analysis, it should be noted that lateral movements of the drilling machine was more likely than an angular movement. Consequently, the values of both coefficients were different and fixed at 0.002 for $\mathrm{a}_{\alpha}$ and at 0.01 for $\mathrm{a}_{\mathrm{r}}$. 


\subsection{Deviation from the reference}

Table 2 and 3 give a summary of the four statistics described above. For the detection of sowing rows and the detection of chicory rows, the trueness and the precision of the method were found to be totally satisfactory for the application, the rows being localized with a trueness of a few centimetres and a good precision. The third quartile was barely higher than the mean, thanks to the asymmetry of the frequency distribution of the deviation. The maximal values are at the limits $(150 \mathrm{~mm}$ for the sowing rows and $50 \mathrm{~mm}$ for the culture rows) or slightly above, which indicate that this limit could sometimes be crossed. The extreme values were often encountered at the beginning and at the end of the line.

The localization of sowing rows seemed more difficult than localization of the chicory rows. The robustness of the neural network used in this later method should however be checked thoroughly.

\section{Conclusions}

The adapted Hough transform was applied on grey level images for the detection of lines or alignments clusters. This method showed one maxima in the Hough space while the classical method gave one local maxima for each line or for each alignment. This maxima presented a good contrast in most conditions.

The method was applied to the detection of seed lines and plant rows. The Hough transform requiring bright lines on a dark background, the images had to be pretreated to reveal the rows or to eliminate unevenness such as shadows. Because of the proximity in the colours of the chicory roots and the soil's colour in one hand, of the wide variability in the lighting on the other hand, it was found difficult to make the crop rows visible in all conditions.

The overall performance of the method, showing a deviation from a reference of few centimetres was found compatible with the application. 


\section{Acknowledgements}

This research was funded by the Walloon Region (Direction Générale de la Technologie et de la Recherche), convention FIRST SPIN off, 011/4797.

\section{References}

[1] F. Pla, J.M. Sanchiz, J.A. Marchant, R. Brivot, Building perspective models to guide a row crop navigation vehicle, Image and Vision Computing, 15(1997) 465-473.

[2] J. A. Marchand, Tracking of row structure in three crops using image analysis, Computers and Electronics in Agriculture. 15 (1996) 161-179.

[3] N. D. Tillett, T. Hague, S.J. Miles, Inter-row vision guidance for mechanical weed control in sugar beet, Computers and Electronics in Agriculture, 33 (2002) 163-177.

[4] Søgaard, Olsen, Determination of crop rows by image analysis without segmentation. Computers and Electronics in Agriculture 38 (2003) 141-158.

[5] F. Rovira-Más, Q. Zhang, J.F. Reid, J.D. Will, Hough-transform-based vision algorithm for crop row detection of an automated agricultural vehicle, Journal of Automobile Engineering 219 (2005), 999-1010.

[6] N.D. Tillett, T. Hague, Computer-vision based hoe guidance for cereals - an initial trial, Journal of Agricultural Engineering Research 74 (1999) 225-236.

[7] T. Pilarski, M. Happold, H. Pangels, M. Ollis, K. Fitzpatrick, A. Stentz, The Demeter system for automated harvesting, Autonomous Robots. 13 (2002) 9-20.

[8] C.M. Onyango, J.A. Marchand, Physics-based colour image segmentation for scenes containing vegetation and soil. Image and vision computing. 19 (2001) 523-538.

[9] V. Leemans, M.-F. Destain, A method to follow Sowing Machine Furrow by using Machine Vision, submitted for publication.

[10] F. van der Heijden, Image Based Measurement System, J. Wiley \& Sons, 1994. 


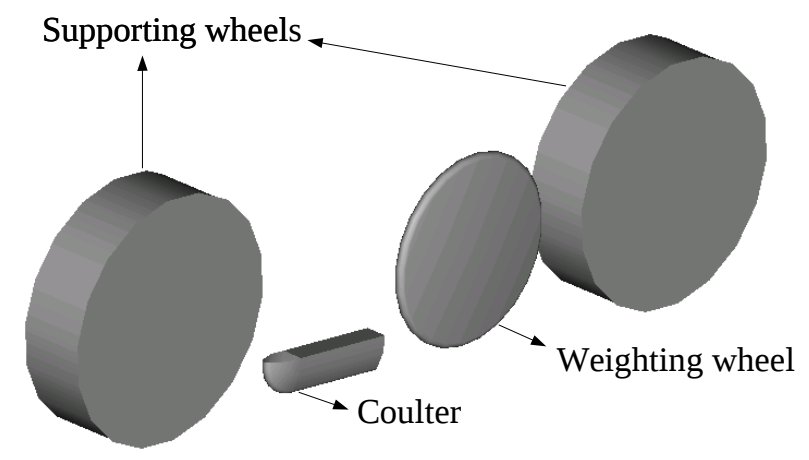

Soil profile of a sowing row

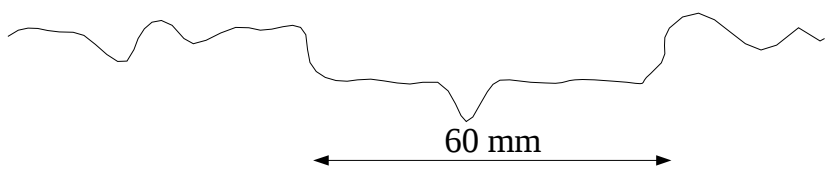

Figure 1: Parts of a sowing machine influencing the transverse profile of a sowing row. The two supporting wheels responsible for a flat track of about $60 \mathrm{~mm}$. The coulter and and the weighting wheel creating a small furrow in the centre of the track. 


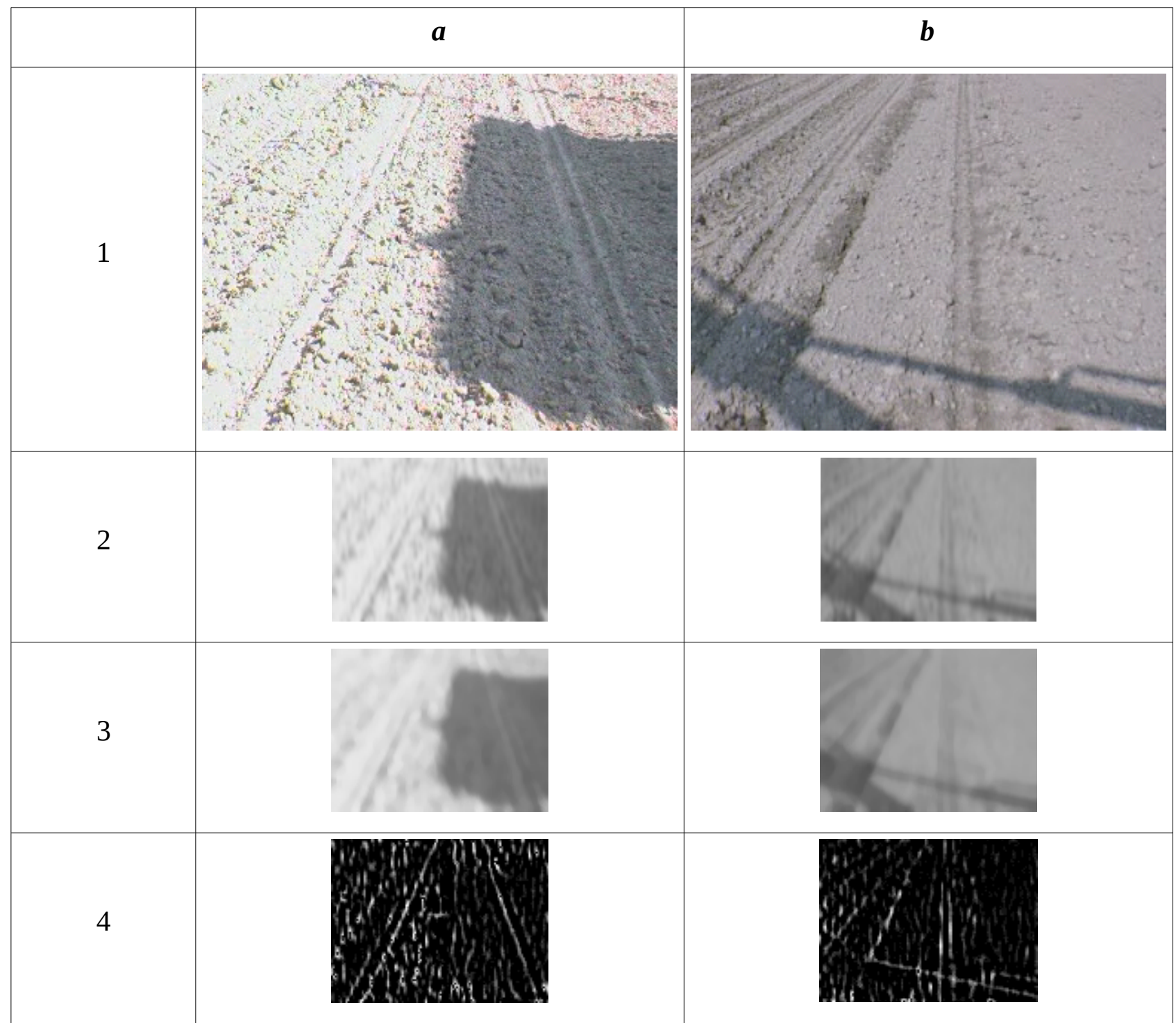

Figure 2 : Pretreatments of the sowing row images.1 : Original images. 2 : images after resizing Gaussian filter. 3 : background images (after median filter). 4 : images after background subtraction. 
Two rows of chicory are visible. The image was acquired under sunny conditions, with the scene in the shade of the machine and sun light reflected by the wind screen of the machine.

Different elements are visible :

- a : chicory root having grown too high over the ground and showing root flesh;

- b : correctly topped chicory

- c : fallen leaf

- d : chicory with leaf stalk

Those two images were extracted from the same video which were acquired under light mist conditions. Settings of the camera and the soil are the same on both images but the illuminant has changed.

Missing plant, plant having grown outside the rows and chicory with long leaf stalk or leaves can be observed. These different elements contribute to reduce the visibility of the plant alignments.
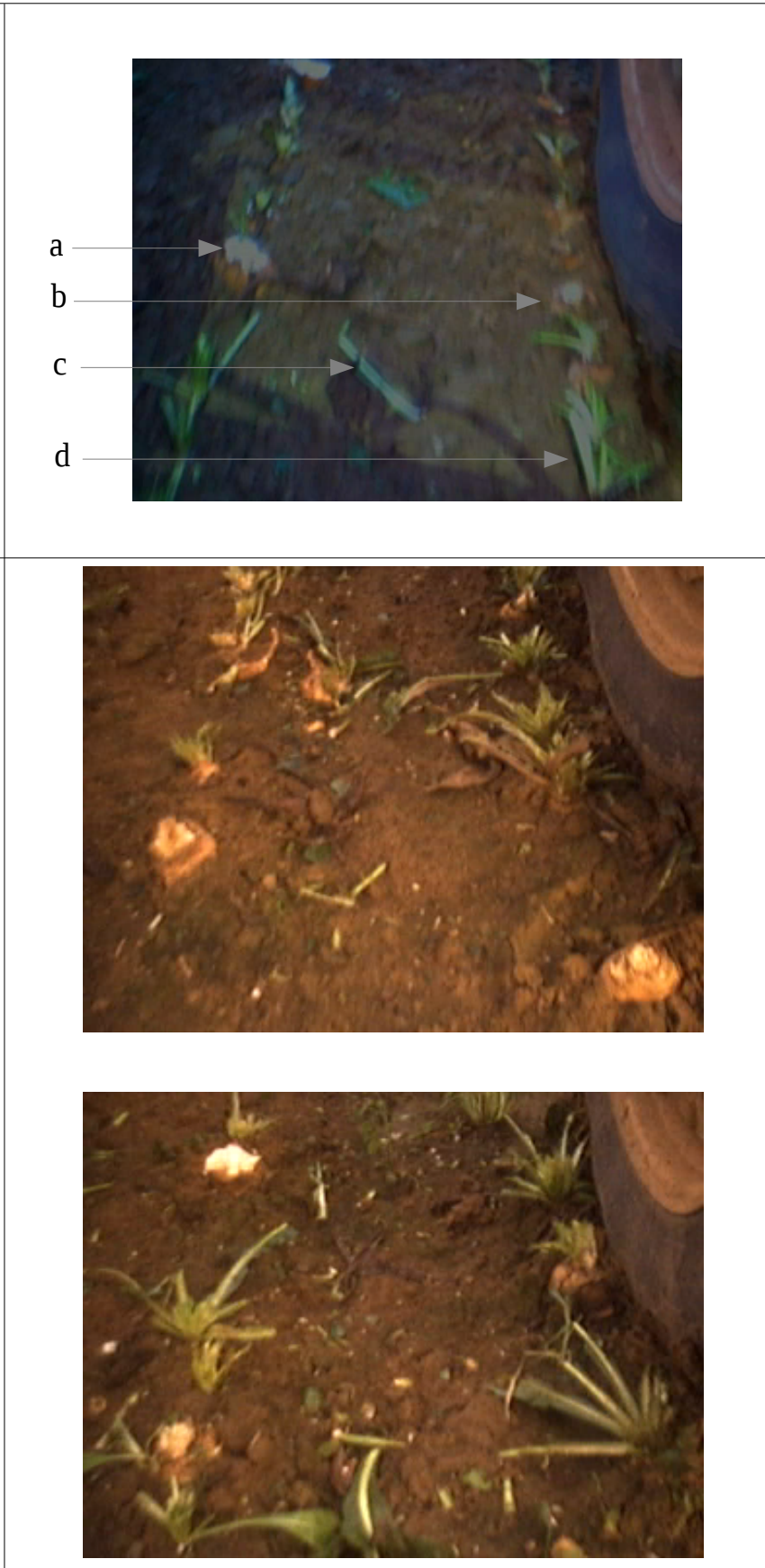

This image was acquired under sunny conditions and showing part of the image in the sun and part in shadow.

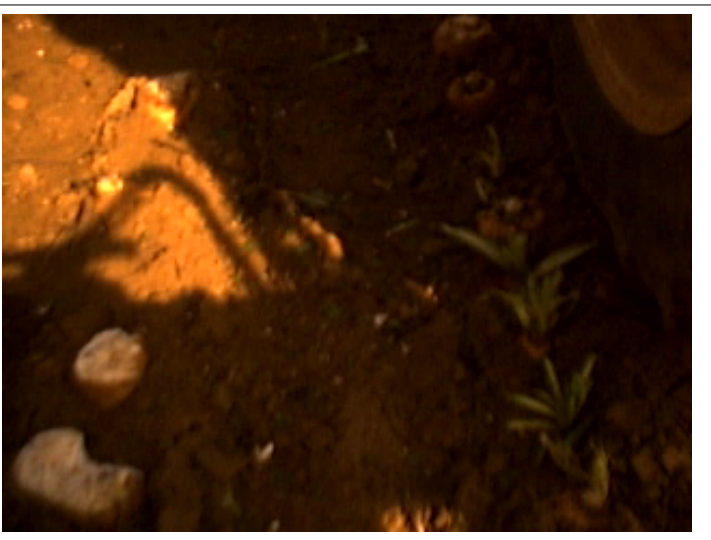

Figure 3: Different elements which could be observed during the harvesting of chicory under different lighting 
conditions.

\section{Classical Hough transform}

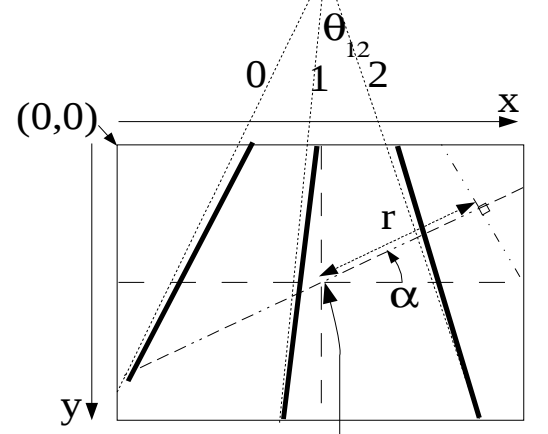

The « Hough space »

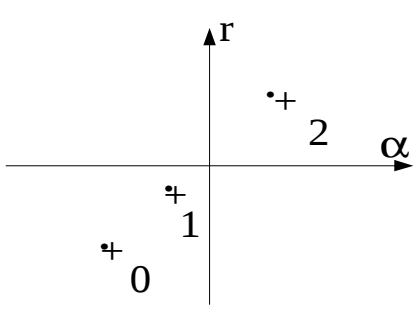

Reference point

: projection line

, + : theoretical position

: integration direction

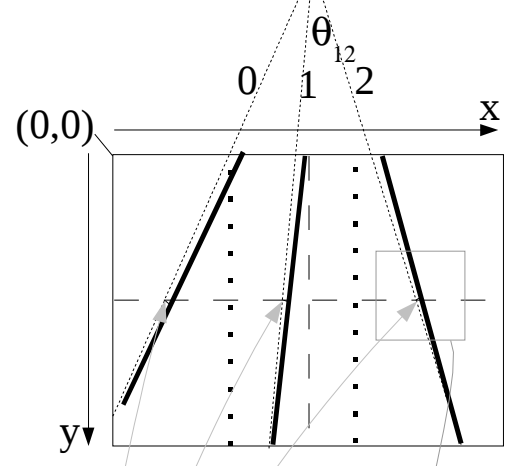

The « Hough space »
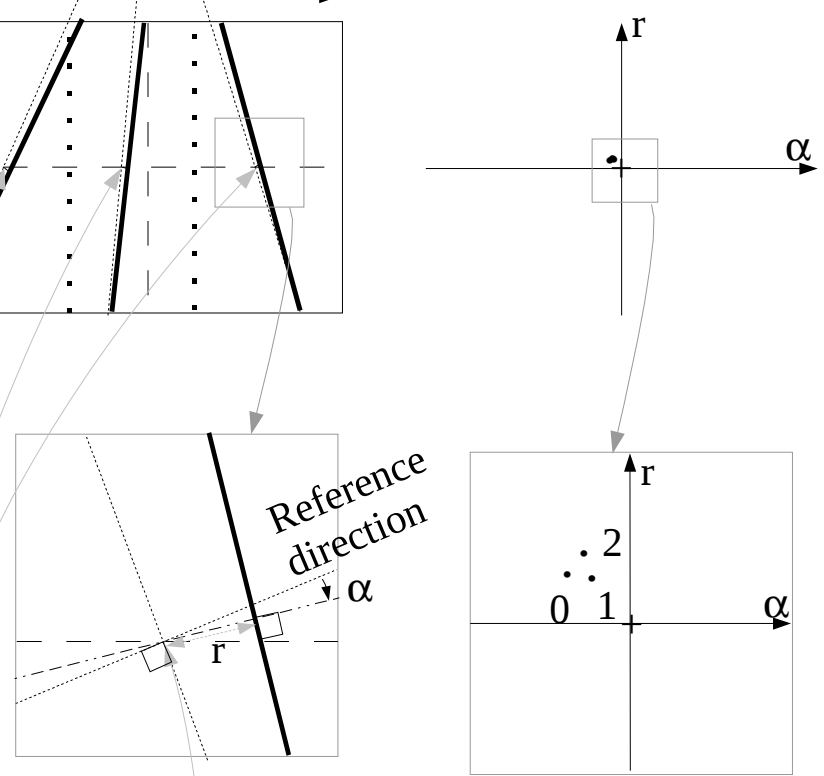

Reference point

: projection line

$+:$ theoretical position ,. : true position 
Figure 4: Principle of the Hough transform adapted to the detection of a line cluster. The classical transform is illustrated above and the adapted one bellow. 


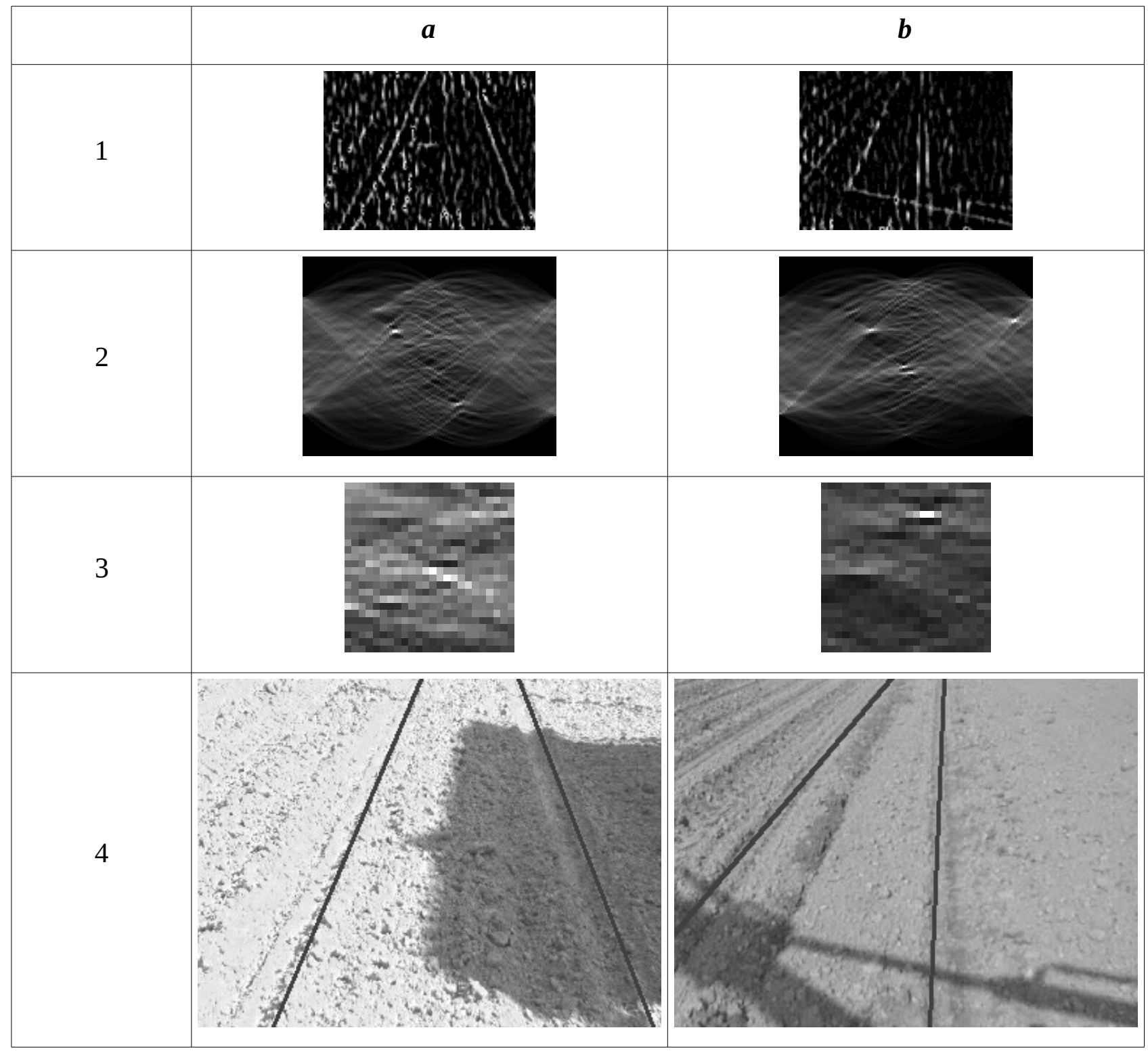

Figure 5: Results of the Hough transform applied to the detection of a cluster of lines - localisation of sowing rows. 1 : Pre-treated images. 2 : Results of the classical Hough transform. 3 : Results of the adapted Hough transform. The grey scale of the images on row 2 and 3 were adjusted so that the minima were represented in black and the maxima in white. 4 : Results of the detection on the original images. 


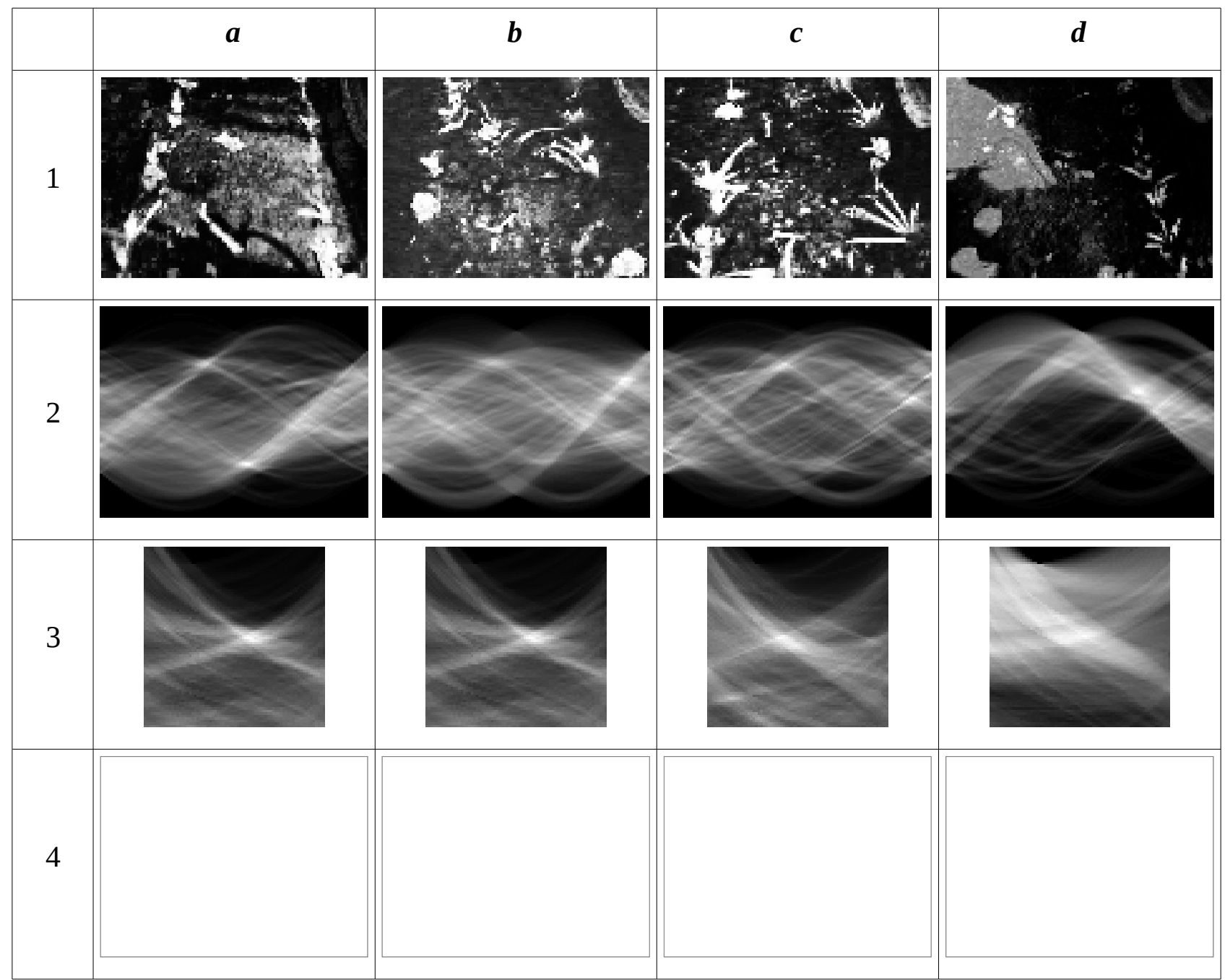

Figure 6: Results of the Hough transform applied to the detection of a cluster of lines - localisation of chicory rows. $1:$ Images treated by neural networks. $2:$ Results of the classical Hough transform. $3:$ Results of the adapted Hough transform. The grey scale of the images on row 2 and 3 were adjusted so that the minima were represented in black and the maxima in white. $4:$ Results of the detection on the original images. 
Table 1 : The number of videos acquired at different places and time.

\begin{tabular}{cccc}
\hline Activity & Year & number of places & number of videos \\
\hline drilling & 2003 & 2 & 19 \\
drilling & 2004 & 4 & 30 \\
harvest & 2003 & 2 & 65 \\
\hline
\end{tabular}


Table 2 : Main statistics of the deviation with the reference concerning the detection of sowing rows. The data are given in $\mathrm{mm}$.

\begin{tabular}{ccccc}
\hline & Trueness (mean) & Precision & Third quartile & Maximum \\
& & $\begin{array}{c}\text { (standard } \\
\text { deviation) }\end{array}$ & & \\
\hline mean & 28 & 22 & 38 & 112 \\
standard deviation & 16 & 14 & 22 & 88 \\
\hline
\end{tabular}


Table 3 : Main statistics of the deviation with the reference concerning the detection of chicory rows. The data are given in mm.

\begin{tabular}{ccccc}
\hline & Trueness (mean) & Precision & Third quartile & Maximum \\
& & $\begin{array}{c}\text { (standard } \\
\text { deviation) }\end{array}$ & & \\
\hline mean & 16 & 6 & 22 & 74 \\
standard deviation & 12 & 5 & 9 & 28 \\
\hline
\end{tabular}

